\title{
La personnalisation d'un tourisme standardisé dans les stations de ski des Alpes du Nord: Opportunités et limites
}

\section{Anouk Bonnemains}

\section{CpenEdition}

\section{Journals}

Édition électronique

URL : http://journals.openedition.org/tourisme/1228

DOI : 10.4000/tourisme.1228

ISSN : 2492-7503

Éditeur

Éditions touristiques européennes

Référence électronique

Anouk Bonnemains, «La personnalisation d'un tourisme standardisé dans les stations de ski des Alpes du Nord : Opportunités et limites », Mondes du Tourisme [En ligne], Hors-série | 2016, mis en ligne le 01 septembre 2016, consulté le 19 avril 2019. URL : http://journals.openedition.org/tourisme/1228 DOI : 10.4000/tourisme.1228

Ce document a été généré automatiquement le 19 avril 2019

\section{cc)}

Mondes du tourisme est mis à disposition selon les termes de la licence Creative Commons Attribution - Pas d'Utilisation Commerciale - Pas de Modification 4.0 International. 


\title{
La personnalisation d'un tourisme standardisé dans les stations de ski des Alpes du Nord: Opportunités et limites
}

\author{
Anouk Bonnemains
}

\section{Introduction}

1 La pratique touristique s'est diffusée dans les sociétés, du tourisme d'initiés vers le tourisme de masse: la démocratisation du tourisme peut être analysée comme la deuxième révolution touristique, mais qu'en est-il actuellement? Les attentes de la clientèle auraient évolué. Le tourisme aujourd'hui se devrait d'être personnalisé, centré sur l'expérience des individus, et associé avec une dimension ludique. Celui-ci s'opposerait à un tourisme dit de masse, basé sur une standardisation des pratiques et des services. Il y aurait donc l'avènement d'un nouveau système touristique (Cuvelier, 1998). Nous soutenons dans cet article que c'est bien à travers un double processus, celui de l'offre et de la demande, que se construisent et se professionnalisent les sites touristiques. C'est bien par l'analyse des trajectoires touristiques qu'il est possible de mettre en avant cette double articulation. Il est pour cela fondamental d'étudier l'ancrage historique des territoires afin de mettre en évidence les dynamiques sous-jacentes à leur évolution. Cela va nous permettre de questionner l'émergence ou non d'un nouveau système touristique. Notre démonstration va s'appuyer sur une région touristique, les stations de ski de haute altitude de Tarentaise. Ce territoire est pertinent dans cette analyse pour deux raisons : 1 . Il s'est construit par l'offre, basant son modèle sur une standardisation des pratiques touristiques. 2. Son modèle socio-économique reste fondé sur une démarche quantitative et une mono-activité (la pratique des sports d'hiver). À partir de là, quelle est la capacité de ce modèle à intégrer un nouveau système touristique ? Il nous faudra définir ce que serait ce nouveau modèle touristique à travers les études sur la demande touristique et les discours des acteurs de ces sites. Nous terminerons sur les trajectoires prises par les stations pour répondre à l'évolution de la demande touristique. 


\section{Les stations de ski de haute altitude de Tarentaise, un modèle fondé sur l'offre}

\section{L'importance socio-économique de ces sites}

2 Les Alpes du Nord françaises, et particulièrement le département de la Savoie avec la vallée de la Tarentaise (carte 1), est l'une des destinations les plus reconnues nationalement et internationalement sur le marché des sports d'hiver. Les stations de ski de la vallée de la Tarentaise font partie des plus grandes stations mondiales (fig. 1). Ces stations ont été pensées dès leur création pour une vocation internationale, ce qui explique leur importance aujourd'hui. À l'exception de Val-d'Isère, les autres stations de sport d'hiver étudiées ont été créées ex nihilo (Knafou, 1978). De manière plus précise, ces stations ont été construites sur des sites vierges habités temporairement l'été pour les alpages.

Carte 1 : carte de localisation de la vallée de la Tarentaise

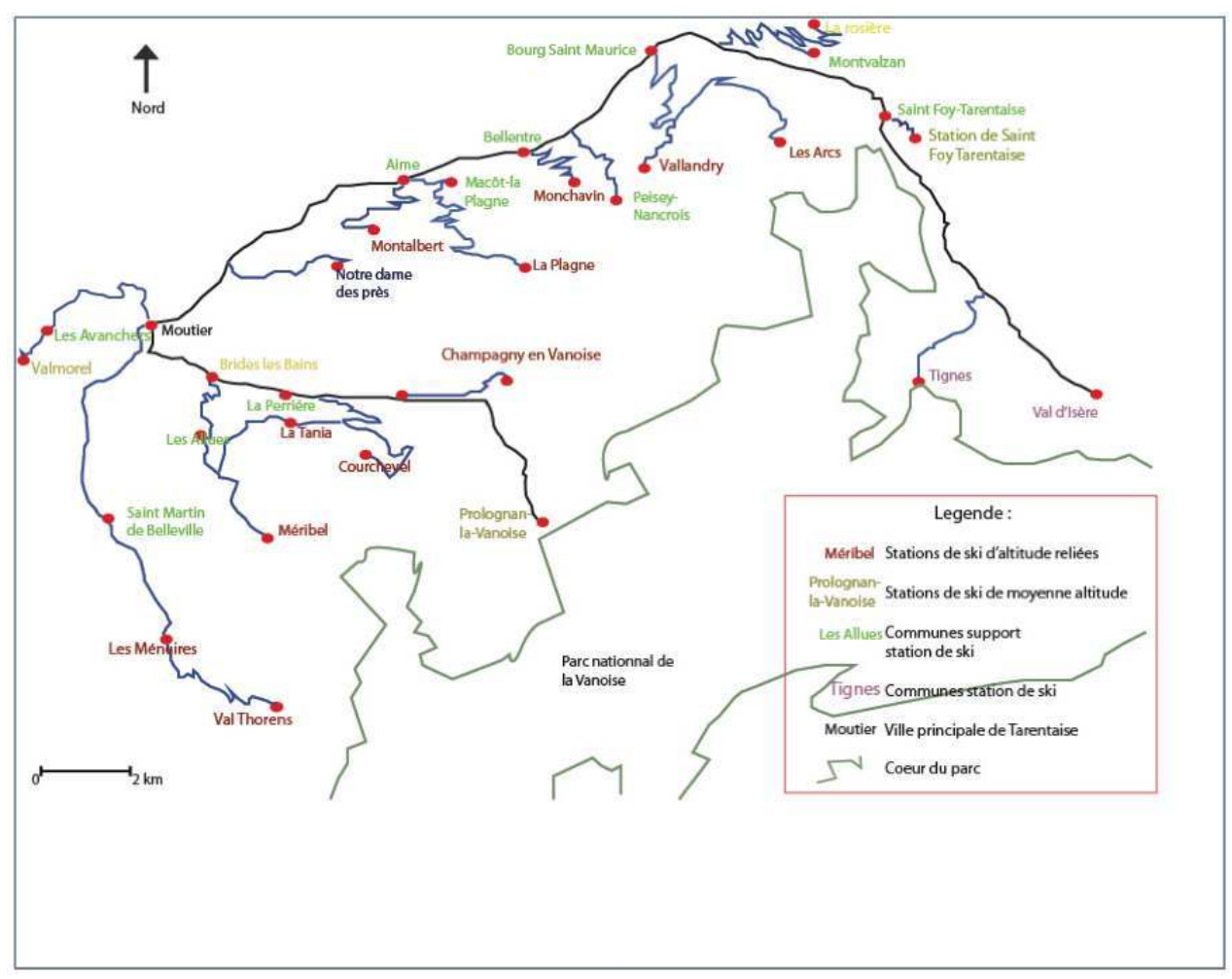

(c) Bonnemains, 2013 .

3 Il semble fondamental de replacer ces stations dans le paysage des sports d'hiver autant au niveau mondial que local, afin de montrer l'importance socio-économique qu'elles représentent pour les populations locales. La vallée de Tarentaise représente $37 \%$ (Domaine skiable de France, 2011) de l'ensemble de la fréquentation touristique des stations de sports d'hiver françaises. La question centrale est de comprendre comment un modèle touristique, implanté à une époque avec toutes les représentations et les préoccupations du moment, a évolué dans le temps, pour rester une véritable industrie, et cherche aujourd'hui à s'adapter à de nouvelles contraintes de personnalisation de 
l'offre. Trois points nous permettent de définir ces sites comme une industrie de service : 1. ils nécessitent un équipement lourd, les remontées mécaniques ainsi que de nombreux aménagements pour les séjours; 2. une division du travail (dans les entreprises de remontées mécaniques...) ; et enfin 3. pour que les installations soit rentables, un nombre important de nuitées doivent être vendues.

Fig. 1 : Le paysage des sports d'hiver français, de l'international à la vallée de la Tarentaise
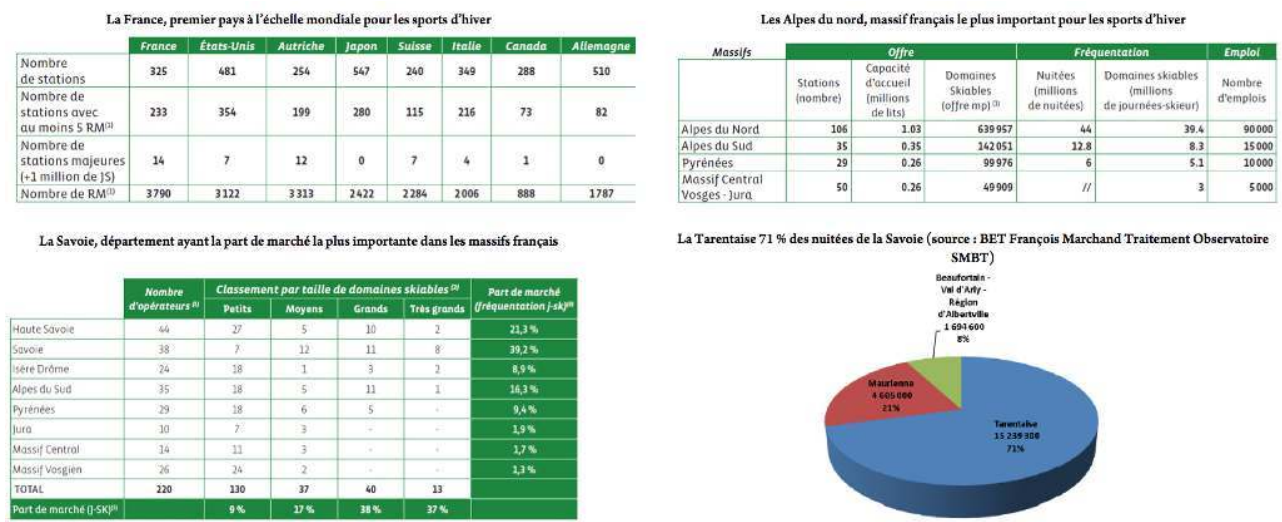

Sources : Domaine skiable de France, 2011.

\section{Le modèle socio-économique de ces stations}

Le modèle socio-économique des stations est basé sur un seuil quantitatif, sous-entendant la prédominance du nombre de touristes, via le taux de fréquentation, pour la rentabilité économique du site. Le modèle économique des stations est resté le même depuis leur création, par contre la place des acteurs dans ce modèle et leur interrelation semblent changer dans le temps.

Fig. 2. Système économique des stations de sports d'hiver de haute altitude de Tarentaise

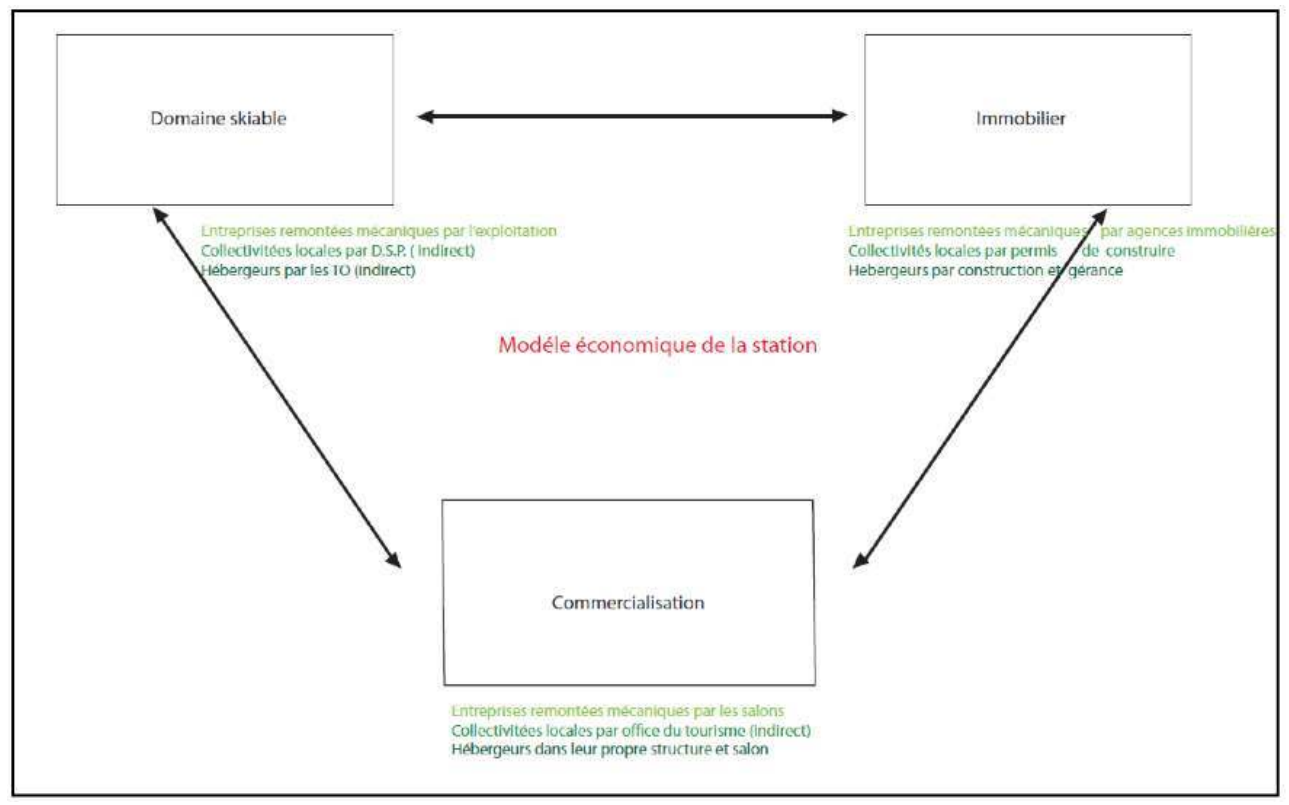

François Fièvre2016-08-22T16:13:00FF@ Bonnemains. 
5 À partir du schéma ci-dessus (fig. 2) nous allons expliciter ce modèle socio-économique : Les trois piliers sont: 1 . Le domaine skiable: la pratique de la glisse est la motivation principale des vacanciers en station de ski, le domaine skiable est donc l'attrait principal pour la clientèle. 2. L'immobilier: les stations de Tarentaise étant éloignées des pôles urbains, ce sont des sites de séjour, d'où l'importance de l'hébergement. 3. La commercialisation: afin d'attirer la clientèle dans leur station, les professionnels ont développé des canaux importants de promotion et de commercialisation du site.

6 À ces trois piliers socio-économiques sont liés trois groupes d'acteurs principaux ayant un impact direct ou indirect sur les piliers identifiés : 1 . Le domaine skiable fait directement référence à l'entreprise de remontées mécaniques, en lien avec la commune par une délégation de services publics pour l'exploitation du domaine skiable. Cette entreprise commercialise le domaine skiable en relation avec l'office du tourisme (lui-même financé pour l'essentiel par la mairie). Et enfin l'entreprise de remontées mécaniques travaille en collaboration avec les tour-opérateurs et les hébergeurs. 2. La commune, concédant son territoire pour l'exploitation du domaine skiable, valide en conseil municipal les éléments importants des entreprises de remontées mécaniques, comme les tarifs. La commune délègue également la promotion et l'animation touristique à l'office du tourisme (soit, en statut association loi 1901, en société d'économie mixte ou en entreprise publique à caractère industriel ou commercial) et donc la commercialisation de la station. Enfin, la commune contrôle les permis de construire à vocation touristique par le biais des plans locaux d'urbanisme, ce qui lui assure un droit de regard sur les constructions immobilières. 3. Les hébergeurs et les tour-opérateurs sont un puissant levier de commercialisation de la station. Les relations entre ces trois piliers sont évolutives dans le temps et dans l'espace, un acteur peut prendre plus d'importance qu'un autre à certaines époques. Un équilibre doit être respecté pour que le système économique reste sain et puisse être durable dans le temps.

\section{La construction de ces sites touristiques}

7 Dès le milieu des années 1960, l'État ambitionne avec le Plan neige de redynamiser les territoires ruraux confrontés à une perte démographique importante. L'État décide, pour un rééquilibrage du territoire français et pour faire face à la concurrence internationale (notamment autrichienne), de se lancer dans un plan de développement des stations de ski. L'État met en place une commission interministérielle de l'Aménagement de la montagne (CIAM, en 1964) pour accélérer la reconnaissance des sites les plus propices à la construction de stations de ski. Rétrospectivement l'État joue donc le rôle d'aménageur des territoires, via la Caisse des dépôts et consignations. C'est tout un imaginaire social associé aux stations de cette génération qui se met en place : celui de la modernité, basée sur une représentation de l'homme et de la nature qui se révélera au travers de l'architecture et de l'urbanisation lors de la phase de construction et de multiplication des aménagements. Cette représentation de la modernité va inspirer l'ensemble des aménagements construits à cette époque. Les architectes ayant travaillé durant cette période sur les stations de ski peuvent être qualifiés de «modernes ». Les stations de ski intégrées de haute altitude deviennent des laboratoires d'expériences pour les architectes.

Finalement, on constate que les architectes et urbanistes projettent une vie nouvelle dans l'organisation de l'habitat de loisir en montagne [...]. Ils tentent à la 
fois de répondre à une attente encore mal identifiée et de produire de nouvelles pratiques. Ces architectes, urbanistes, designers, sont tous des modernes. Ils ont créé les stations dans l'esprit du temps: fonctionnalisme, rationalité, etc. Ils s'inscrivent dans les réflexions autour de l'Homme Nouveau, qui pour la première fois intègre le loisir et la détente dans son quotidien. (Wozniak, 2002, p. 21)

C'est une vision fonctionnaliste et standardisée des vacances pensée autour de la pratique des sports d'hiver en direction d'un public citadin qui se met en place sur le territoire de la Tarentaise. Il y a une volonté tacite de démocratisation du ski, d'où l'exploitation touristique massive ayant pour conséquence une standardisation de la pratique. Les stations de sports d'hiver continuent de se développer en multipliant les aménagements ; cette dynamique est renforcée par les départs au ski qui s'accroissent, avant de stagner à partir du milieu des années 1980. La nécessité d'une structuration et d'une organisation des stations en terme de destinations touristiques se fait de plus en plus sentir. De plus, c'est à partir de cette époque qu'une combinaison de phénomènes (sociaux, climatiques et financiers) montre les premières limites du développement des stations de ski. La stagnation des taux de fréquentation, liée à un taux de départ aux sports d'hivers ne dépassant pas les $8,5 \%$, malgré les $20 \%$ venté par le SEATM (Wosniak, 2002), va accentuer la concurrence nationale et internationale entre les stations, l'offre devenant plus importante que la demande touristique. La concurrence est également présente avec les autres destinations touristiques à l'échelle mondiale. Il y a là le passage d'un développement uniquement basé sur l'offre vers une prise en compte grandissante de la demande touristique. Ce qui va nous amener à réfléchir à ce que serait aujourd'hui la demande touristique: peut-on parler de "l'avènement d'un nouveau système touristique »?

\section{Quelles représentations de la demande touristique?}

9 Il est possible de définir la demande touristique comme la revendication des attentes des individus lors de leurs séjours touristiques. Le point de départ de notre démarche portait sur la caractérisation des besoins actuels des touristes dans les études sur la demande touristique. Pour cela l'examen de 19 études relatant les attentes actuelles des individus lors de séjours touristiques a permis d'identifier des caractéristiques communes et des évolutions. Ces études sont de natures différentes: sur la demande touristique d'une manière générale (4 études), sur les attentes des touristes vis-à-vis de la montagne, et les attentes vis-à-vis des sports d'hiver (14 documents). Elles s'échelonnent entre 1993 et 2011. L'identification des études s'est faite par rapport aux différentes échelles: du local au national, ensuite entre les études portant sur la demande touristique globale vers la spécificité de cette demande en montagne et en stations de ski. Certains documents relatent le profil de la clientèle (l'âge, le sexe, CSP, origine...), alors que d'autres cherchent à mettre en exergue les motivations des individus à partir en vacances. En parallèle, d'autres rapports, en cherchant les motivations des touristes, définissent les grandes tendances actuelles de la consommation afin de donner une opérationnalité marketing (les «carnets de route de la montagne » d'Atout France, les «Savoie Mont Blanc Tourisme ») sous-traitant avec des bureaux d'études (TNS-Sofres, SocioVisonCofremca...). Et enfin les études universitaires portent particulièrement sur l'expérience touristique. 
10 Pour bien comprendre comment ces études sont révélatrices des évolutions sociétales, il est important de les replacer dans leur contexte historique. La première étude sur la clientèle en station de sports d'hiver date de 1987 avec Sema Metra (Wosniak, 2002). Il est intéressant de constater que :

Le postulat de base [de cette étude] est que la clientèle potentielle est homogène. L'étude reprend donc l'idée que seuls des skieurs composent la clientèle des stations d'altitude, alors même que L. Chappis proposait, dès 1974, une typologie des clientèles «ski ». La conclusion de l'étude sera ainsi orientée par ce postulat. (Wosniak, 2002, p. 11.)

11 Au fil des études sur la demande touristique, celle-ci est perçue de manière de plus en plus complexe, jusqu'à questionner la personnalisation et l'expérience touristique comme l'avènement d'un nouveau système touristique.

\section{L'expérience touristique}

12 Le tourisme actuel est défini comme opposé au tourisme de masse standardisé, celui-ci ne répondant pas à l'injonction actuelle de recherche d'autonomie et de réalisation de soi (Kaufmann, 2004) ; en effet le tourisme doit permettre à l'individu de se révéler à luimême. L'expérience touristique aurait pour vocation de donner du sens, de la valeur à ce que vit l'individu (Frochot et Kreziak, 2011), c'est une recherche d'émotion. L'expérience touristique se fonde sur trois aspects :

1. Elle est partagée, puisque «l'être ensemble», la convivialité et le partage d'émotions au sein du groupe, à différents niveaux, sont au cœur de la satisfaction des touristes. 2. Elle se construit dans une collaboration entre les touristes et la station, dont le rôle est à la fois de leur éviter les écueils et de leur proposer un éventail de services avec lesquels ils créent la valeur de leur séjour, dans un processus de co-construction. 3. Enfin elle se vit dans la durée, puisque les touristes s'immergent dans l'expérience de service pendant plusieurs jours, au cours desquels le ski et l'état mental de déconnexion en résultent, le « flow » joue un rôle central. (Frochot et Kreziak, 2009, p. 11.)

13 L'expérience touristique se construit en partie sur la personnalisation de l'offre touristique, puisque l'une des caractéristiques de la société postmoderne (Chevallier, 1994) ou de la «société liquide» (Bauman, 2013), est l'individualisme. Les vacances seraient aujourd'hui orientées vers l'exceptionnel et le développement de soi, d'où la recherche d'un tourisme personnalisé. Les nouvelles technologies ont joué un rôle central dans cette personnalisation de l'offre touristique ; notamment c'est par le biais d'Internet que les clients cherchent l'offre qui leur convient le mieux, dans l'idée d'un séjour surmesure. «La demande de personnalisation de l'offre qui caractérise aujourd'hui les rapports des consommateurs à la production et à la distribution. » (DGCI, 2010, p. 82.) La personnalisation de l'offre touristique serait une volonté de la part de la clientèle d'échapper à la massification et à la banalisation. C'est l'envie d'être considéré dans la spécificité de ses besoins, afin de renforcer son autonomie et sa réalisation individuelles.

Le second élément participant à l'expérience touristique est la notion de coproduction du séjour entre la clientèle et les professionnels du tourisme.

En devenant plus autonome et plus mature, le consommateur, à l'opposé d'un consommateur passif, devient de plus en plus un consommateur "coproducteur " de l'offre, c'est-à-dire partie prenante active de la démarche de conception et de production de l'offre. (DGCI, 2010, p. 19.) 


\section{matériel, l'alimentation ainsi que la segmentation de la clientèle : jeunes, gay, famille.} peuvent participer de ce mouvement de « coproduction ».

Les clients auraient une exigence accrue envers la qualité des prestations touristiques, notamment par l'augmentation du confort et l'importance des coûts engagés pour partir en vacances. Ils auraient également de plus en plus conscience de leurs droits et de leur pouvoir économique. De nombreuses réflexions sont soulevées par cette question de l'exigence de la clientèle. A-t-elle toujours existé de la part des populations les plus fortunées? Se serait-elle diffusée à d'autre catégories de population? "Accumulant des expériences variées de touristes et de clients de loisirs, ils ont un "univers de référence" aux standards de plus en plus élevés. ( (DGCI, 2010, p. 26.) En allant plus loin dans la réflexion, certains auteurs ont développé le concept de culture touristique personnelle :

la culture touristique personnelle [...] nous espérons réhabiliter définitivement l'expérience touristique comme fait culturel majeur de plusieurs styles de vie tout à fait caractéristique de notre époque. Du point de vue culturel, le tourisme ne se limite plus à quelques escapades au cours d'une année pour se changer les idées et changer d'air. Il est intégré à la vie et à la culture de l'homme moderne. (Laplante, 2006, p. 103.)

Est-ce une évolution du tourisme de masse ou une remise en cause de ce tourisme ? L'expérience touristique ne peut être effective sans un élément central, celui de la rupture avec le quotidien.

\section{Rupture avec le quotidien}

Les vacances ont une fonction de rupture avec le quotidien, de ce fait le lieu touristique doit permettre à l'individu de s'immerger complètement dans ce moment de détente et de retrouvailles familiales. La rupture avec le quotidien se concrétise à travers la mobilité, on part de chez soi pour se rendre dans un autre lieu qui est par opposition hors du quotidien. Il ressort, d'après l'étude d'Isabelle Frochot et Dominique Kreziak (2011) sur l'expérience touristique en station de montagne, un besoin de distanciation avec la vie quotidienne urbaine, même si très souvent la description des vacances en station de sport d'hiver est une transposition assez fidèle de la ville urbaine à la montagne. La clientèle des stations de Tarentaise est majoritairement citadine. La montagne serait un antidote à la ville que les clients souhaitent quitter momentanément. Cette rupture s'incarne notamment dans l'envie de ne pas utiliser la voiture, dont l'emploi est très mal perçu dans la mesure où elle ne facilite pas «l'immersion » et le dépaysement attendu lors du séjour. La notion de convenience, au sens de fluidité des services, devient donc un élément central pour la satisfaction de la clientèle. De plus, pour que l'immersion soit complète, l'offre touristique se doit d'être diversifiée, simplifiée afin de rassurer et de donner des certitudes à la clientèle.

Au global, le rôle de la station est d'assurer la fluidité du service, en apportant un éventail d'activités sur la base desquelles les clients vont construire leur propre expérience et leur propre vécu de consommation. (Frochot et Kreziak, 2009, p. 10.)

La rupture avec le quotidien permet de renforcer un des aspects essentiels de l'expérience touristique, l'immersion dans le moment présent. Les études en marketing du tourisme mettent l'accent sur la nécessité de la part des professionnels du tourisme de prendre en compte et de développer cet élément. En station de sports d'hiver, le ski permettrait aux pratiquants de connaitre le flow, l'état d'esprit d'une personne quand elle est 
complètement absorbée dans l'instant présent. Celui-ci renforce le sentiment de dépaysement et de coupure avec le quotidien. Le ski offrirait également la possibilité « de résoudre une partie des tensions vécues par les individus dans leur propre vie et des formes sociales valorisées dans la société » (Frochot et Kreziak, 2011, p. 27).

20 Selon les études analysées les touristes auraient actuellement une forte sensibilité au ludique, aux activités permettant la convivialité. Le ski toute la journée ne serait plus recherché, la demande touristique se tournerait vers une "vitalité soft ». Ces éléments renforceraient la rupture avec un quotidien synonyme de rapidité, de compétition et de performance. L'orientation actuelle des activités irait vers la contemplation, le farniente accentuant la sensibilité aux lieux, aux ambiances. La recherche actuelle du bien-être et de la santé auraient pour finalité la remise en forme pour le retour à la vie quotidienne.

Le séjour au ski est décrit à travers le champ sémantique de la remise en forme, comme un grand bol d'air pur, et les termes de fraîcheur, de pureté, d'oxygénation ou d'absence de pollution sont très fréquemment utilisés. Il est considéré comme un moyen efficace et rapide de se remettre en forme et de repartir plus efficacement vers la vie quotidienne. (Frochot et Kreziak, 2009, p. 27).

21 Ces éléments renforcent l'importance de la qualité et du caractère esthétique de l'environnement dans lequel les touristes viennent passer leur séjour. Sur les stations de sports d'hiver, la rupture et le dépaysement s'incarnent en partie dans le mythe des sports d'hiver. Les personnes se rendant dans un lieu touristique ont un imaginaire sousjacent. En ce qui concerne la montagne, les chalets, la neige sont autant de représentations que les individus cherchent lors de leurs séjours, même si la réalité ne correspond pas. L'imaginaire construit par les individus autour de la destination est fondamental, puisqu'il va en partie déterminer le choix du lieu des vacances, la satisfaction qu'ils vont en retirer et enfin le discours qu'ils vont véhiculer à leur retour.

Selon ces études, l'acte d'achat d'un séjour touristique ne doit pas être négligé, puisqu'il implique beaucoup le touriste, autant financièrement que mentalement. Les clients auraient le souci d'être rassurés et conseillés, d'où le besoin de services. Aujourd'hui les valeurs décrites jusqu'ici seraient ancrées, d'ailleurs les touristes répondraient aujourd'hui en terme de " désir d'être » (être en famille...) et non en désir de faire (faire du ski...). Une réflexion sur l'expérience touristique doit prendre en compte aussi le besoin de retrouvailles avec la famille et les amis.

\section{Retrouvailles avec la famille}

Selon le groupe de recherche mené par Jean Viard « Réinventer les vacances, la nouvelle galaxie du tourisme ", il y aurait deux motivations principales pour partir en vacances : l'une centrée sur l'individu (nous l'avons abordée) et les retrouvailles en famille ou entre amis. L'expérience touristique doit être partagée, la dimension relationnelle devient fondamentale dans une société ou les liens sociaux sont devenus faibles (Bauman, 2013), c'est une recherche de nouvelles sociabilités. Celle-ci se concrétise dans la demande de relations humaines directes. "La question des temps et des lieux de vacances comme moment fort du ressourcement familial et amical ne s'est pas démentie loin de là.» (Viard, 1998, p. 98.)

24 Les vacances seraient devenues le mode d'organisation du temps social, notamment par rapport à la famille. C'est lors des vacances et du tourisme que peuvent se construire ou se reconstruire les solidarités familiales. L'accélération du temps (Gwiazdzinski, 2011), 
notamment dans les zones urbaines, renforce la nécessité de prendre un temps en commun, celui-ci se cristallisant autour des vacances et des loisirs. C'est un autre rapport au temps qui est souhaité lors des vacances, où l'individu serait en capacité de le maîtriser.

Ce que nous venons d'évoquer n'est pas sans contradictions, notamment entre les discours et les pratiques. L'intérêt du travail réalisé est de mettre en exergue les éléments centraux de la demande touristique à travers ces études afin d'identifier ou non l'avènement d'un nouveau système touristique.

Le marketing est devenu un élément important de nos sociétés de consommation; la concurrence entre les destinations touristiques, corrélée à une maturité du marché, pousse les acteurs des stations à rentrer dans des stratégies marketing.

\section{Le marketing}

Une très grande importance est donnée au marketing pour le développement du tourisme en montagne.

L'utilité d'un vrai marketing professionnel pour développer la montagne. 1. Pour se développer, pour développer leurs chiffres d'affaires et consolider leurs positions dans des marchés touristiques de plus en plus concurrentiels, le marketing est aujourd'hui utile, voire nécessaire, aux différents acteurs de la montagne. 2. Le marketing, c'est un état d'esprit couplé à un ensemble de techniques précises et pointues qui ont largement fait leurs preuves et qui s'adaptent très bien au tourisme de montagne, mais qui nécessitent des investissements conséquents, déterminés et professionnels. 3. Le marketing touristique pour la montagne implique souvent de travailler ensemble, regroupé, avec les villages, vallées et massifs d'à côté afin d'exister comme destination au plan régional, national et international. (Afit, 2000, p. 74.)

Les outils marketing sont utilisés comme réponse à un marché qualifié de mature avec des taux de départ au ski qui stagnent; du coup comment renouveler la clientèle ? D'une manière plus précise, c'est l'orientation vers un marketing différencié qui est prônée dans cette étude pour faire face aux nouvelles problématiques des stations de montagne :

Un marketing touristique différencié pour séduire, convaincre et fidéliser des clients très divers et élargir la clientèle de montagne été et hiver. 1. Pour développer les chiffres, il est aujourd'hui conseillé de sortir de la pensée unique. Familles, seniors, juniors, proximité, différentes clientèles étrangères, autant de clientèles qui ont chacune des attentes particulières et légitimes. 2. Le marketing touristique développe des techniques qui permettent de choisir ses clientèles et de répondre à chacune de façon spécifique et adaptée (produit, prix, mise en marché, communication) et enfin d'imaginer la cohabitation entre elles. (Afit, 2000, p. 75.)

Nous allons maintenant regarder comment ces études sur l'avènement d'un nouveau système touristique sont prises en compte dans les discours des acteurs des stations, et quelles sont leurs répercussions sur les sites touristiques. 


\section{Les stratégies d'adaptation à la demande touristique : analyse discursive des représentations des acteurs des stations de ski mises en regard de leur application}

La demande est devenue le moteur des évolutions des destinations touristiques, servant de légitimation pour la plupart des aménagements.

\section{Les représentations des acteurs sur la demande touristique}

Les acteurs s'accordent sur l'idée d'une exigence accrue de la clientèle dans la qualité des services et des activités proposés. Pour répondre à cette demande, on voit se développer une scénarisation des domaines skiables (snowpark, espace ludique, etc.), et une augmentation des animations pour l'ambiance de la station et pendant le moment du ski. Selon les acteurs, ce serait un reflet des évolutions sociétales, de ce qu'ils nomment la maturité du consommateur, ou la culture touristique personnelle. Cette exigence va de pair avec une montée en gamme de l'immobilier.

On a une montée de gamme donc une attente, une exigence de la clientèle. Ça revient à ce qu'on disait toute à l'heure, l'offre, il y a de plus en plus d'offres donc on ne peut plus se contenter de dire il y a de la neige, donc il faut faire du service pour faire de la qualité, ça c'est clair qu'au niveau des pratiques il y a une vraie connaissance du client [...], j'entends par là des tables de pique-nique, avant les gens prenaient leurs sandwichs et s'asseyaient sur un rocher dans la neige. (Entretien, station de Tignes, 2012.)

L'évolution technologique tient une part importante dans ces mutations. Sur le domaine skiable, les remontées mécaniques permettant de monter plus haut plus rapidement et le travail des pistes facilitant la pratique du ski apparaissent comme des évolutions positives. L'évolution dans la consommation du ski est très souvent discutée, puisque c'est l'activité majeure de ces stations. Tous s'accordent à dire qu'il y a une "sousconsommation » du ski aujourd'hui par rapport à une ou deux décennies en arrière. De manière plus précise, les touristes ne skieraient pas moins qu'avant, mais moins que ce que l'équipement permettrait. Il y a le constat d'un suréquipement qui s'expliquerait par la recherche d'un meilleur confort, engendrant beaucoup de conséquences économiques puisque cela demande des investissements très importants.

Les RM vont plus vite, sont plus confortables, les pistes sont plus larges, mieux préparées, le matériel a aussi évolué. (Entretien, Val-d'Isère, 2012.)

On a des clients qui font le même dénivelé qu'il y a 20 ans, pareil ils prennent le même nombre de RM ou un peu moins, parce qu'en général on les fait de plus en plus longues. Mais globalement le dénivelé en hectomètre monté est le même qu'il y a 20 ans, pareil. La seule chose qui diffère c'est qu'avant il leur fallait 8 heures pour le faire et qu'aujourd'hui en 5 heures c'est fait. Il n'y a plus la queue, vous avez de très grosses « catapultes » qui vous emmènent très fort très loin, et donc du coup le temps qui était perdu à la montée s'est considérablement réduit. Il y a donc une fatigue qui arrive plus vite dans la journée. (Entretien, La Plagne, 2012.)

Les discours sur la consommation du ski reflètent assez bien les évolutions sociétales décrites en première partie de ce travail. Les domaines skiables doivent être ludiques et " fun », permettant aux clients d'essayer plusieurs zones dédiées. Il y aurait également une demande importante d'aménagements du type table de pique-nique ou banc pour la contemplation. Selon de nombreux professionnels du tourisme en station, les personnes 
n'auraient plus goût de l'effort, ils souhaiteraient juste faire un ski tranquillement, s'amuser et contempler. En d'autres termes, les acteurs du tourisme, pour maintenir les taux de remplissage, sont allés chercher cette clientèle en lui faisant des skis, des pistes, des remontées mécaniques de plus en plus confortables. L'expérience touristique est centrale dans les discours des acteurs, elle est abordée principalement par la question de l'animation et du ludique. Selon eux, les vacances aux sports d'hiver doivent être "fun ", de là découle l'importance de la convenience. La « vitalité soft » est aussi mise en avant par les professionnels $\mathrm{du}$ tourisme, notamment par l'importance qu'auraient pris la contemplation et le besoin de bien-être, qui n'est pas contradictoire avec l'importance du ludique puisque les deux peuvent aller de pair. Le plus gros bouleversement viendrait de la généralisation d'Internet, bouleversant autant la commercialisation de la station (par l'importance des sites Internet et des réseaux sociaux), que les modes de réservation des logements. Ce changement pousse les acteurs des stations à s'adapter à ces transformations et à offrir de nouveaux services.

C'est bien à moi, office du tourisme, de mettre en place un accueil numérique qui te rende service, qui soit géolocalisé, qui arrive à comprendre tes attentes, qui arrive à comprendre tes besoins. (Entretien avec un directeur d'office du tourisme, Val Thorens, 2012.)

Par voie de conséquence de nouveaux métiers se développent, il y a une nécessité à suivre ces évolutions pour rester compétitif. La demande de personnalisation de l'offre est très souvent abordée lors des entretiens, elle est vécue comme une nouvelle injonction principalement liée aux nouvelles technologies. Cette recherche de personnalisation est aussi perçue par les acteurs à travers les nouveaux processus d'information, particulièrement Internet (même si le bouche-à-oreille reste la principale source d'information), et à travers les prix, par la recherche du prix le plus bas et du «bon plan ». Les acteurs insistent sur ce qu'ils nomment le «juste prix» du séjour, offrir un séjour de qualité au prix qu'il vaut. Il ne faut pas gonfler les prix, car le ski est déjà un sport élitiste et cher. Les réservations se feraient de plus en plus tardivement, la clientèle chercherait des forfaits à la carte poussant les professionnels du tourisme à développer des produits de plus en plus personnalisés.

La place du relationnel est souvent interrogée dans les entretiens, les sports d'hiver seraient des vacances principalement familiales ou amicales, où chaque individu aurait des moments seul ou en groupe de niveau concernant la pratique du ski, et des temps de sociabilité familiale ou amicale (fin de journée, soirée...). On peut voir l'évolution de ces sociabilités à travers l'évolution des grilles tarifaires des remontées mécaniques, un des exemples révélateurs est celui de la création du forfait tribu (réduction du prix du forfait à partir de trois ou quatre adultes).

L'environnement est interrogé principalement sous l'angle du paysage dans les discours, celui-ci tiendrait un rôle important pour la clientèle dans la rupture avec le quotidien. La qualité du paysage hivernal est essentiellement discutée par les professionnels du tourisme par la réduction du nombre de pylônes pour les remontées mécaniques, et même du nombre de remontées mécaniques qui permet également d'optimiser l'exploitation. Enfin le dernier point que nous voulons souligner est l'importance du marketing dans les discours des acteurs des stations de sports d'hiver rencontrés : la quasi-totalité des entretiens se sont rapidement orientés vers le marketing.

Tignes est une marque, Tignes doit être gérée comme une marque. (Entretien,

Tignes, 2012.) 
On a mis en place une politique de positionnement, de repositionnement marketing je dirais, le positionnement en deux mots, en partant d'un constat client voir quelle place on veut occuper dans leur esprit, c'est une bataille de perception. (Entretien, Val-Thorens, 2012.)

Dans chaque station étudiée, des services marketing ont été ouverts dans les offices du tourisme et entreprises de remontées. Un nombre important d'acteurs mettent en avant la création de ces "nouveaux métiers", aujourd'hui toutes les sociétés de remontées mécaniques comme les offices du tourisme (hors la Tania) du territoire de recherche ont un service ou une personne responsable du marketing. Cet aspect de la professionnalisation des stations, à travers les acteurs, est très souvent explicité au cours des entretiens.

Nous avons construit un schéma synthétique afin de mettre en lien les discours des acteurs et les trajectoires prises par les stations (fig. 3), nous allons particulièrement insister sur deux éléments, la diversification des activités et la différenciation entre les stations.

Fig. 3. Lien entre les représentations des acteurs de stations de sports d'hiver de haute altitude de Tarentaise et les actions entreprises

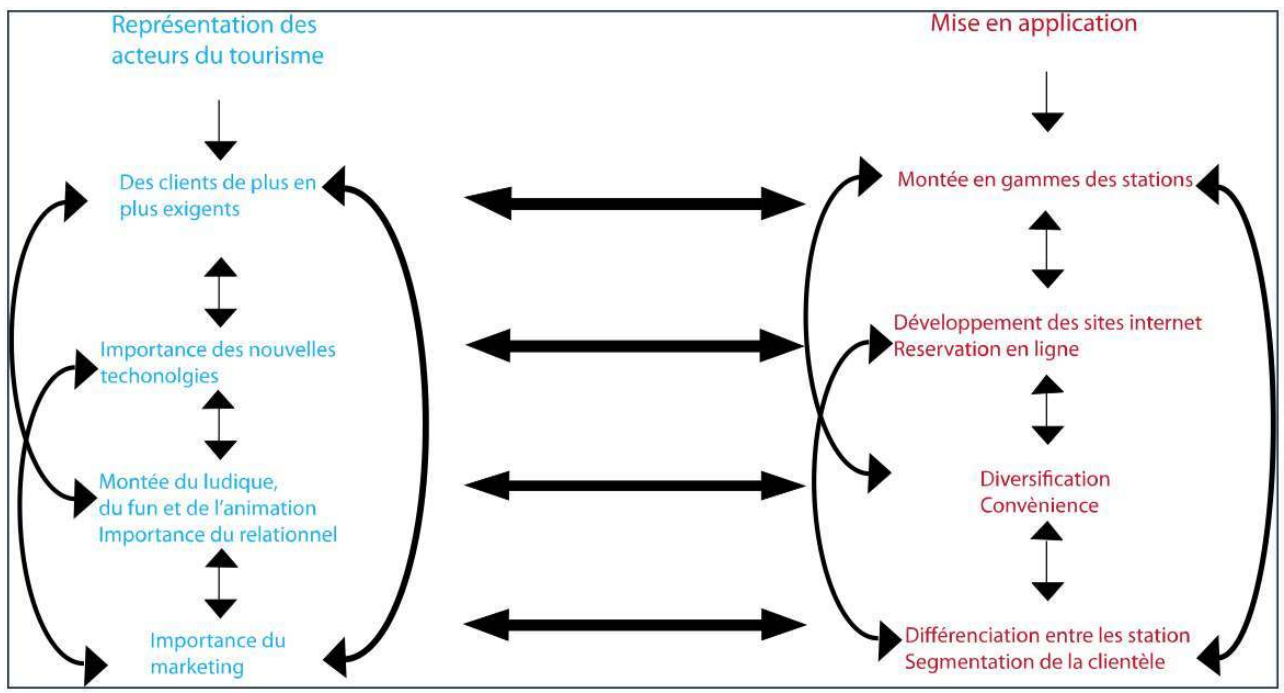

(c) Bonnemains.

Les stations de ski de haute altitude de Tarentaise sont dans une dynamique de montée en gamme (Marcelpoil, 2007), les nouveaux aménagements construits ou rénovés tel que l'hôtellerie sont dans un processus d'amélioration continue de l'offre. La montée en gamme se ressent dans la plupart des projets de ces sites touristiques. La diversification des activités et la différenciation entre les stations peut participer de ce mouvement. Au cours de cet article, l'importance du développement des sites internet a déjà été abordée, il n'en sera pas question dans cette partie. 


\section{Le choix de la diversification des activités et de la différenciation entre les stations}

\section{La diversification du système touristique}

40 La diversification des activités dans les stations de ski est une partie importante de l'évolution des stations, même si les acteurs s'accordent sur la centralité du ski comme motivation première pour un séjour en station. Il nous faut définir plus précisément ce qu'est la diversification: en fonction de quoi les stations de ski la mettent en place. Si la réponse est une diversification par rapport à une pratique du ski standardisée, tous les nouveaux aménagements sur les domaines skiables rentrent dans la diversification. Par contre, si c'est se diversifier par rapport à la pratique des sports de glisses, seuls les nouveaux aménagements tels que les centres aqualudiques, ou les pistes de luge sur rail (aux Ménuires) rentrent dans la diversification.

41 Deux types de diversification se dégagent: 1 . Une diversification par rapport à une pratique du ski standardisé: scénarisation de certaines pistes, zones dédiées aux débutants, à la pratique du ski ou du snowboard free style... 2. Une diversification au sein de la station: centre aqualudique, animation, piétonnisation... mais qui reste perçue comme un complément au ski.

Le produit initial c'est le ski, mais quand vous choisissez votre hôtel pour aller sur la plage regarder s'il y a l'eau, une piscine, ça c'est le minimum vous allez voir s'il y a des toboggans pour les enfants, s'il y a bien des animations pour les enfants, si c'est du tout compris ou pas du tout compris, s'il y a une navette qui peut vous emmener au village pour pouvoir faire quelque chose. C'est des petits extras que nous exploitants il y a 20 ans on n'avait pas à se préoccuper de ça, mais aujourd'hui c'est ça qui fait la différence. (Entretien avec un directeur d'entreprise de remontées mécaniques.)

Sur le domaine skiable, l'émergence de nouvelles pratiques va demander de nouveaux aménagements. L'apparition des sports postmodernes va faire évoluer en partie le domaine skiable.

Le sport moderne intègre aux valeurs du passé la spécificité de son époque, à savoir la compétition, qui se concrétise par la rénovation des Jeux olympiques à la fin du XIX ${ }^{\mathrm{e}}$ siècle et présuppose la mise en place de structures spécialisées, notamment les clubs, les associations, les comités et les fédérations. Par opposition, l'image du sport postmoderne porte l'empreinte de la société qui le produit. La société de consommation et de loisir, qui découle des progrès techniques et technologiques, de l'amélioration des conditions de vie et de la réduction du temps de travail, a modifié le paysage sportif par l'accroissement et la diversification des pratiques. (Lacassagne et alii, 2006, p. 4).

La mise en place de snowparks, zones dédiées à la pratique «free style ", va faire évoluer petit à petit le domaine skiable vers un espace ludique. Ces lieux où se pratiquent de nouvelles formes de glisse demandent des aménagements et du matériel spécifiques, ils vont se développer dans toutes les stations, devenant un critère important de choix notamment pour une clientèle de jeunes hommes sportifs :

L'éclectisme et la dispersion qui caractérisent le comportement sportif contemporain depuis les années 1970 reflètent la quête « d'aspects ludiques et de libre expression » qui se substituent aux principes de rationalité prédominant dans le sport moderne. (Lacassagne et alii, 2006, p. 5.) 

d'abord standardisée pour devenir accessible au plus grand nombre, va par la technologie se diversifier. Certaine zones dédiées à la pratique du ski «moderne », comme les stades de slalom, continuent d'exister; par contre d'autres ont été remplacées. L'exemple du kilomètre lancé (KL) est révélateur : à son apogée aux Jeux olympiques de 1992 avec une piste olympique aux Arcs, il ne reste plus que celle-ci dans la Tarentaise alors que dans les années 1990 beaucoup de stations en possédaient un. Les zones dédiées vont devenir des espaces ludiques, qui vont se multiplier sur les domaines skiables. Ces zones correspondent au ciblage de la clientèle fait par les entreprises de remontées mécaniques. Si le positionnement marketing de la station souhaite faire venir une clientèle familiale, il sera mis en place des pistes à thème pour les enfants avec des aménagements spécifiques. Il en est de même si l'on cherche à attirer une clientèle jeune et sportive : le snowpark aura une place importante dans les investissements de l'entreprise. Néanmoins, ces zones dédiées ne sont pas exclusives, elles cohabitent sur les domaines.

Ces zones deviennent importantes également du fait que la consommation du ski, au sens du nombre d'heures passées sur le domaine skiable, est de moins en moins importante. C'est un discours récurent des professionnels de la montagne. C'est à partir de là que la station de sports d'hiver prend toute sa place en tant que destination touristique, et cherche elle aussi à se diversifier pour offrir un complément à l'activité ski. Cette ouverture est bien à comprendre comme un complément à l'activité ski, il n'est en aucun cas question de rendre le produit ski secondaire, bien au contraire il s'agit de le renforcer par de nouvelles attractivités. Ces nouveaux aménagements sont légitimés par l'adaptation à la demande touristique et aux nouveaux types de consommation du ski, les clients ne passeraient plus 8 heures sur les pistes, il faut donc leur proposer de nouvelles activités.

Fig. 4a. Vue du centre aquatique de Courchevel

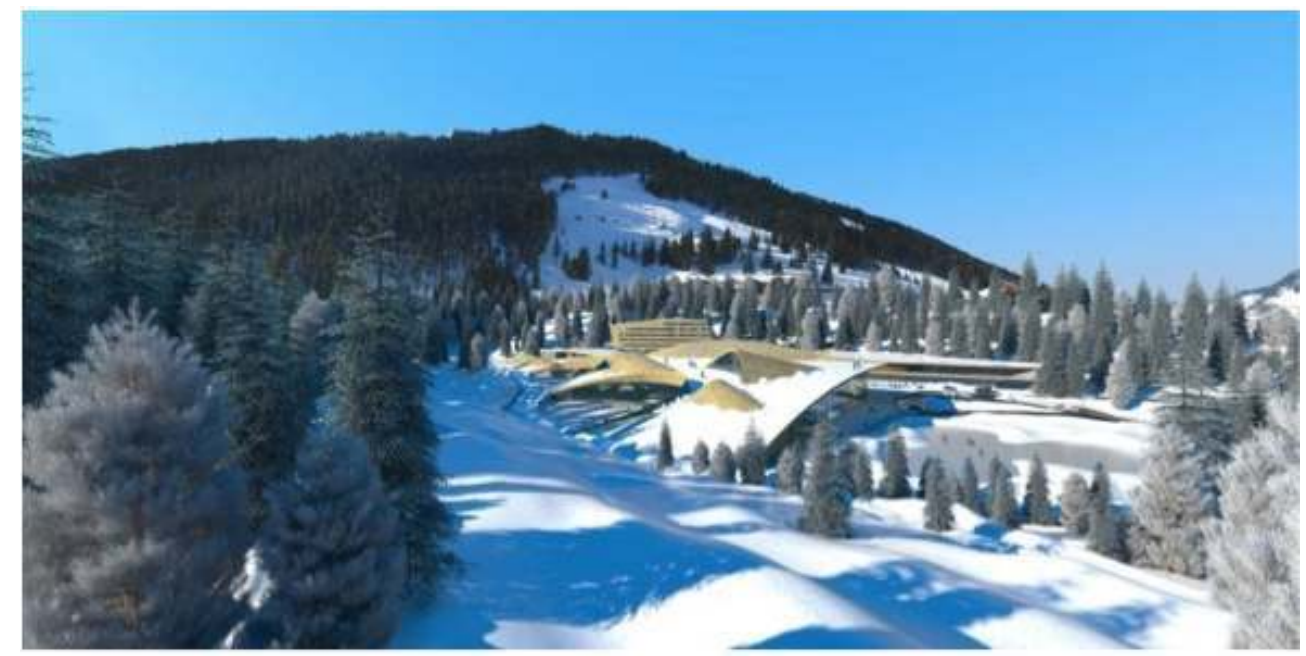

Source : site web de la mairie de Courchevel, consulté le 19 août 2014 
Fig. 4b. Vue du centre aquatique de Courchevel

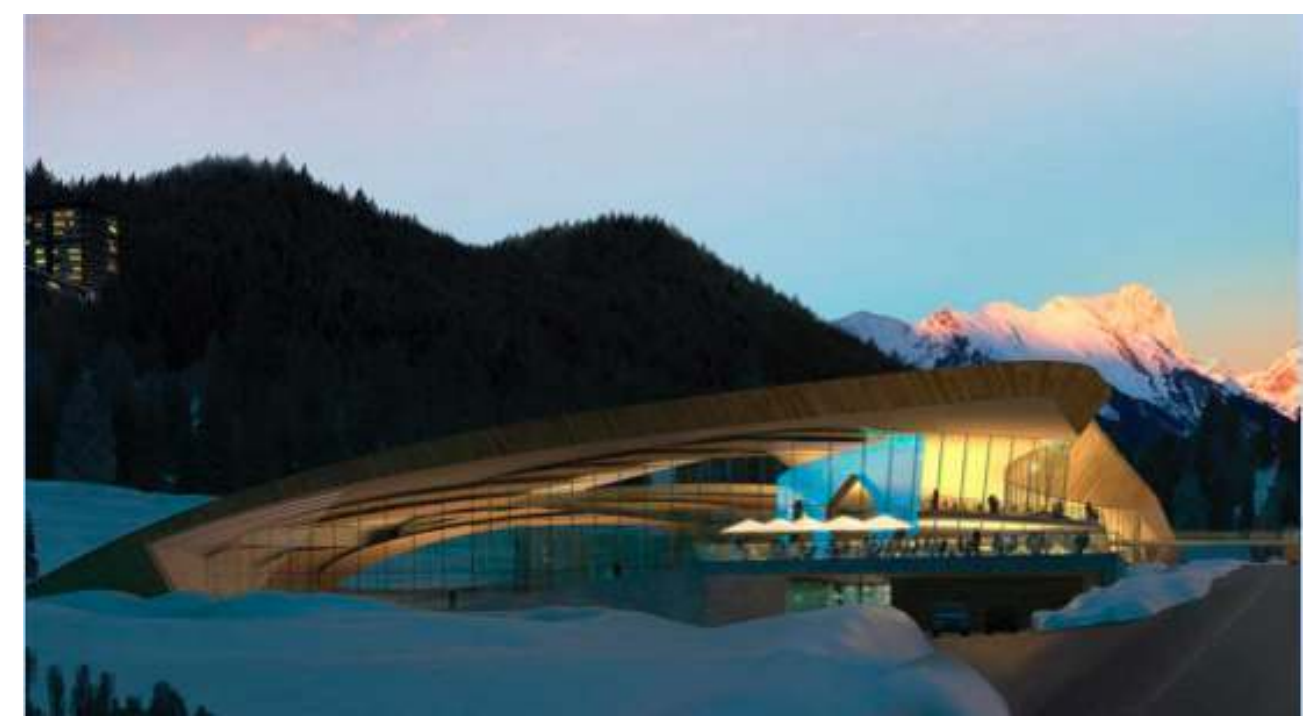

Source : site web de la mairie de Courchevel, consulté le 19 août 2014.

Les figures $4 \mathrm{a}$ et $4 \mathrm{~b}$ montrent l'architecture futuriste du projet du centre aquatique de Courchevel qui s'est ouvert début 2016. Cet espace représente :

$1300 \mathrm{~m}^{2}$ de surface d'eau ( $1000 \mathrm{~m}^{2}$ en intérieur, $300 \mathrm{~m}^{2}$ en extérieur), $9600 \mathrm{~m}^{2} \mathrm{de}$ surface couverte (centre aquatique et restaurants), $10500 \mathrm{~m}^{2}$ d'espaces aménagés, $160 \mathrm{~m}^{2}$ de surface de vente ( 4 boutiques) pour un cout de $63 \mathrm{M}$. d'euros $\mathrm{HT}^{1}$.

Il est bien entendu que toutes les stations de sports d'hiver ne se lancent pas dans de tels projets, cependant chaque station souhaite en avoir un. Ce serait devenu un critère de choix pour les touristes, mais c'est également un «jeu» de rapport de force entre les stations. Ces aménagements extrêmement coûteux et pas forcement rentables sont légitimés : 1. par l'image qu'ils donnent à la station; 2. par l'attrait de la clientèle vers le bien-être; 3. par la complémentarité entre les deux saisons afin de valoriser la saison d'été. La question qui se pose est l'endettement important des communes pour mener à bien ces projets et entretenir ces équipements.

En ce qui concerne les autres formes que pourrait prendre la diversification, elles restent des "niches", au sens d'activités touchant une minorité de personnes: comme les raquettes, les courses de chiens de traineau, les pistes de luge... Il nous semble difficile de parler de diversification touristique, puisque ces aménagements et ces activités ne sont pas là pour diminuer la place du domaine skiable; bien au contraire, ils sont là pour renforcer son attrait. C'est par la différenciation que les stations souhaitent rester attractives.

\section{La différenciation entre les stations}

Les sports d'hiver sont un marché mature et très concurrentiel, il y a donc un enjeu important quant à la concurrence, entraînant des logiques de différenciation entre les stations. Cette logique doit permettre au territoire d'être plus attractif et de renouveler sa clientèle. Cette préoccupation, due au vieillissement de la population venant faire du ski et à la difficulté de faire venir de nouvelles clientèles, est liée à l'évolution des pratiques touristiques. 
50 Les logiques de différenciation sont d'autant plus fortes que les stations de sports d'hiver se sont équipées d'un nombre important d'aménagements, tels que des centres aqualudiques et des pistes de luge, standardisant l'offre. Cela permet de mieux comprendre les logiques de différenciation qui vont de pair avec des logiques de mimétisme (Bertin, 2011). Il est d'ailleurs tout à fait possible de mettre en avant différentes étapes dans ces logiques de standardisation (mimétisme) et de différenciation. 1. La première façon de se distinguer a été apportée par le kilométrage de piste, en étendant le domaine skiable vers le haut ou en se reliant à d'autres domaines. La majorité des grandes stations ayant un fort pouvoir d'investissement ont créé des domaines reliés, ce qui a en partie standardisé l'offre de ski mais pas celle de séjour. 2. Ces sites touristiques se sont équipés de nouveaux aménagements pour se différencier de leurs concurrents, logique de mimétisme entraînant une standardisation de l'offre. 3. Le marketing est l'outil privilégié des professionnels du tourisme des stations de sports d'hiver, celui-là permettant la différenciation de celles-ci. Le marketing permet également, via les nouvelles technologies, la personnalisation de l'offre touristique :

Les applis iPhone si tu veux se développent [...], et que si je ne t'envoie pas une information qui peut te rendre service par rapport à tes gouts, si le soir je t'envoie «puch » en te disant : « N'oublie pas ce soir concert de musique classique ou de jazz viens nombreux. » Là je t'ai rendu service, et moi de toute manière je le paye le service donc autant que ce soit plein. Après qu'avec ton téléphone tu prennes des photos et que tu les balances sur Facebook ou sur Youtube peu importe. Comme la moitié des personnes qui sont en vacance, tu postes tes photos pendant tes vacances et en plus ça va influencer les autres personnes plus tard pour venir chez toi. (Entretien avec le directeur de l'office du tourisme de Val-Thorens.)

51 Cette citation nous montre l'importance des nouvelles technologies, mais également l'influence du « bouche-à-oreille 2.0 ». La station de Tignes est révélatrice d'une stratégie marketing poussée vers les nouvelles technologies et un public de jeunes sportifs, puisque la station ne produit plus de brochure papier, tout passe par Internet et les applications.

\section{Conclusion}

52 Ces trajectoires, ainsi que la "nouvelle demande touristique ", s'incarnent particulièrement dans la problématique de la rénovation du bâti. L'exemple des Arcs est en ce sens révélateur des dynamiques des stations de sports d'hiver: en 2002 débute la création d'Arcs 1950 avec une architecture pseudo néorurale correspondant aux attentes actuelles de la clientèle. En effet, les stations sont confrontées à un enjeu de taille, la rénovation du bâti. L'adaptation à la demande touristique se cristallise autour de cette question. Les appartements construits lors de la création de ces stations ne correspondent plus aux critères actuels : comment faire en sorte que ces sites restent attractifs? Ces opérations de réhabilitation, et les projets toujours actuels de construction de nouvelles résidences de tourisme ou hôtels, sont argumentés à nouveau à partir de l'évolution des attentes de la clientèle. Les anciens appartements ne sont plus estimés conformes aux attentes de la clientèle actuelle, le modèle fonctionnaliste standardisé que l'on pourrait qualifier de «fordiste» ne serait plus ce que recherche la clientèle d'aujourd'hui. Les pratiques touristiques ont évolué et avec elles les besoins en services, en qualité d'accueil et d'hébergement.

53 Nous conclurons sous forme de questions, puisque ce travail appelle à de nombreuses interrogations. Comment penser la résilience d'un système touristique basé sur du 
volume, de la fonctionnalité et de la standardisation face aux injonctions actuelles à la personnalisation, à la coproduction et à l'expérience touristique? Les stations de sports d'hiver étudiées ont fait face à de nombreuses perturbations depuis leur création : prise de conscience d'une diminution de la fiabilité de l'enneigement, difficulté dans la commercialisation des appartements. Elles ont jusque-là trouvé des parades : techniques avec la neige de culture, commerciales avec la montée en gamme des stations. Ces sites touristiques ont donc fait preuve d'une capacité d'adaptation. Jusqu'à quel point peuventelles s'adapter? Ne vont-elles pas être contraintes spatialement, par le cadre bâti et naturel?

Peut-on aujourd'hui parler d'un tourisme personnalisé ? Est-ce une remise en question de l'ancien système ou une évolution de celui-ci sans réelle transformation? Qu'est-ce qui pousserait les acteurs à repenser un système qui fonctionne? Pour les acteurs du tourisme le système fonctionne, puisque les stations de sports d'hiver restent rentables, voire très rentables.

\section{BIBLIOGRAPHIE}

Agence française de l'ingénierie touristique (Afit), Carnet de route de la montagne : de l'écoute des clients à l'action marketing, Afit, 2000.

Zygmunt Bauman, La Vie liquide, Pluriel, 2013.

Erik Bertin, «Le vertige de la différenciation, tropisme de la pensée stratégique dans le champ du marketing et de la communication? ", Communication, vol. 28/1, 2011, p. 101-132 [https:// communication.revues.org/2056].

Marc Chevallier, Les Sports d'hiver, espaces et culture : généalogie culturelle des représentations et usages touristiques hivernaux en montagne, thèse de géographie, université de Grenoble, 1994.

Pascal Cuvelier, Anciennes et Nouvelles Formes de tourisme. Une approche socio-économique, L'Harmattan, 1998.

Direction générale de la compétitivité de l'industrie et des services (DGCIS), Nouvelles Perceptions de la valeur des offres touristiques: Impacts pour les opérateurs, 2010.

Domaine skiable de France, Recueil indicateurs et analyses 2011, 2011 [http://www.domainesskiables.fr/fr/publications/observatoire/].

Isabelle Frochot et Dominique Kreziak, «Étude de l'expérience touristique en stations de montagne, hiver et été », étude réalisée pour Savoie Mont Blanc Tourisme, 2009.

Isabelle Frochot et Dominique Kreziak, « Co-construction de l'expérience touristique. Les stratégies des touristes en stations de sport d'hiver ", Décisions marketing, n 64, 2011, p. 23-33.

Luc Gwiazdzinski, «Temps et territoires : les pistes de l'hyperchronie », Territoires 2040, $\mathrm{n}^{\circ} 6$, p. 75-97 [http://territoires2040.datar.gouv.fr/spip.php?article221].

Jean-Claude Kaufmann, L'Invention de soi. Une théorie de l'identité, Armand Colin, 2004. 
Rémy Knafou, Les Stations intégrées de sports d'hiver des Alpes françaises : l'aménagement de la montagne à la « française ", Masson, 1978.

Marie-Françoise Lacassagne, Léone Pizzio et Ahmed Jebrane, « La représentation sociale du sport : vision d'étudiants sportifs et non-sportifs », Movement \& Sport Sciences. Science \& Motricité, $\mathrm{n}$ o 58, 2006, p. 117-134.

Marc Laplante, L'Expérience touristique contemporaine. Fondement sociaux et culturels, Presses de l'université du Québec, 1996.

Emmanuelle Marcelpoil (dir.), Nouvelles Pratiques touristiques en zones de montagne : vers un renouvellement des pratiques de gestion foncière?, Direction du Tourisme, 2007.

Jean Viard (dir.), Réinventer les vacances. La nouvelle galaxie du tourisme, La Documentation française, 1998.

Marie Wozniak, « Les stations de ski : quelles représentations des clientèles pour quel cadre bâti ?", Revue de géographie alpine, vol. 90, nº 4, 2002, p. 17-31.

\section{NOTES}

1. Site web du centre aquatique de Courchevel, consulté le 19 août 2014 [ http:// www.centreaquatique.mairie-courchevel.fr/fr_FR/chiffres-cles/].

\section{RÉSUMÉS}

Il serait question aujourd'hui de l'avènement d'un nouveau système touristique qui se révélerait à travers la coproduction de l'expérience touristique entre les professionnels et la clientèle. Quelle est la genèse de ce nouveau système touristique? Comment s'incarne-t-il sur un territoire? Dans cet article nous souhaitons apporter des pistes de réflexion sur ces nouveaux enjeux en se basant sur une région touristique : la vallée de Tarentaise, en Savoie, cœur du tourisme hivernal. Nous avons analysé diverses études sur la demande touristique ainsi que les discours des acteurs du tourisme recueillis lors d'une enquête de terrain. Est-ce que les acteurs de cette zone touristique identifient les mêmes enjeux que les auteurs des études sur la demande touristique? Quels sont les liens entre ces études et les discours? Nous pouvons mettre en avant une relation étroite entre ces deux éléments, notamment à travers les trajectoires d'adaptation à la clientèle prise par les stations.

It would question today the advent of a new system tourist who would prove through coproduction the tourist experience between professionals and clients. What is the genesis of this new tourism system? How if he plays in a territory? In this article we wish to bring lines of thought on these new stakes by basing itself on a tourist region: Valley Tarentaise, Savoie, the heart of winter tourism We have analyzed various studies on tourism demand and the discourse of tourism stakeholders gathered in a survey of territory Are the actors in this tourist area identify the same issues that the authors of studies tourism demand? What are the links between 
these studies and the speech? We can highlight the close relationship between these two elements, in particular through the paths Customer adaptation taken by the stations.

INDEX

Mots-clés : système touristique, sports d'hiver, demande touristique, trajectoire d'adaptation des territoires

Keywords : tourism system, winter sport, tourism demand, territories adaptation path

\section{AUTEUR}

\section{ANOUK BONNEMAINS}

Doctorante, laboratoire Environnement, dynamique et territoires de montagne (EDYTEM), UMR 5204, université de Savoie - bâtiment « Pôle Montagne » F-73376 Le Bourget du Lac anouk.bonnemains@univ-savoie.fr 